\title{
Cerebellar fastigial nucleus is involved in post-stroke depression through direct cerebellar-hypothalamic GABAergic and glutamatergic projections
}

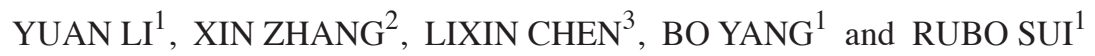 \\ ${ }^{1}$ Department of Neurology, The First Affiliated Hospital of Jinzhou Medical University, \\ Jinzhou, Liaoning 121001; ${ }^{2}$ Department of Pharmacy, General Hospital of Fushun Mining Bureau, Fushun, \\ Liaoning 113008; ${ }^{3}$ Nursing College of Jinzhou Medical University, Jinzhou, Liaoning 121001, P.R. China
}

Received April 6, 2018; Accepted July 27, 2018

DOI: $10.3892 / \mathrm{etm} .2019 .7913$

\begin{abstract}
The present study aimed to investigate whether the cerebellar fastigial nucleus (FN) is involved in post-stroke depression (PSD), and to observe the effect of direct cerebellar-hypothalamic $\gamma$-aminobutyric acid (GABA)ergic and glutamatergic projections on PSD, in order to understand the mechanisms underlying the cerebellar modulation of mood and emotion. Healthy Sprague-Dawley rats were randomly divided into five groups: Sham-operated, Stroke, PSD, FN lesion, and decussation of superior cerebellar peduncle (XSCP) lesion groups. Sham surgery was performed in animals of the Sham group ( $n=6)$. The rats in the other four groups ( $\mathrm{n}=6$ for each group) underwent middle cerebral artery occlusion. The rats were examined twice a week in an open field test. In addition, the expression of cytokines in hippocampal tissues, and the content of glutamate and GABA in the lateral hypothalamic area (LHA) were measured. The results showed that scores corresponding to the behavioral signs of depression were decreased in the PSD, FN lesion and XSCP lesion groups. In addition, the mRNA levels of tumor necrosis factor- $\alpha$, interleukin (IL)- 6 , and IL- $1 \beta$ in the hippocampus of the PSD, FN lesion and XSCP lesion groups were significantly increased. The GABA and glutamate content in the LHA were also decreased significantly in the PSD, FN lesion and XSCP lesion groups. Taken together, the findings of the present study indicated that the cerebellar FN may be involved in PSD through the direct cerebellar-hypothalamic glutamatergic and GABAergic projections.
\end{abstract}

Correspondence to: Dr Rubo Sui, Department of Neurology, The First Affiliated Hospital of Jinzhou Medical University, 2 Renmin Street, Jinzhou, Liaoning 121001, P.R. China

E-mail: suirubo0123@163.com

Key words: post-stroke depression, fastigial nucleus, lateral hypothalamic area, direct cerebellar-hypothalamic projection

\section{Introduction}

Post-stroke depression (PSD) is one of the most frequent psychiatric complications of stroke and affects $\sim 33 \%$ of stroke patients (1). Patients who have suffered a stroke who also suffer from PSD only show minor improvement in rehabilitation compared with stroke patients without depression. Therefore, it is necessary to investigate its mechanisms.

The majority of current PSD theories consider PSD to be caused by underlying problems, including hippocampal neuronal regeneration disorder, the presence of inflammatory cytokines, hypothalamic-pituitary-adrenal axis disorder, monoamine deficiency, and neuroanatomical problems, for example, frontal lobe neuronal injury. Among these, the presence of inflammatory cytokines has been linked to the underlying mechanisms of PSD and several studies have shown that the elevation of cytokines is a key factor in the development of nerve cell damage leading to PSD $(2,3)$. However, the specific mechanism underlying the elevated cytokines remains to be fully elucidated.

Increasing evidence has suggested that the cerebellum may be important in cognition, behavior, and psychiatric illnesses $(4,5)$. Schmahmann and Sherman found isolated cerebellar lesions in patients, which can lead to cerebellar cognitive affective syndrome (CCAS) (4). In addition, a key feature of CCAS is the development of dysregulation when the cerebellar fastigial nucleus (FN) is involved in the lesions (6). Evidence from one of our previous studies showed that depressive-like behaviors in PSD rats were improved by FN electrical stimulation (FNS) (7). Similarly, Su et al reported that electrical stimulation at the FN substantially alleviated symptoms in patients with PSD (8). Taken together, there is a possibility that cerebellar dysfunction may be involved in the underlying mechanisms of PSD. However, few studies have investigated the putative role of the cerebellum in PSD.

A previous study indicated that stimulation of the cerebellar FN can provide a protective effect by inhibiting cerebrovascular inflammation (9). In addition, lesions of neuronal somas in bilateral FN with kainic acid (KA) have been shown to lead to the enhanced activity of T cells, B cells and natural killer cells (10). Our previous preliminary study also showed that, 
following FNS, the expression of inflammatory cytokines in the hippocampus was reduced significantly, thus protecting the Purkinje cells in this region and simultaneously improving the depressive-like behaviors of the PSD rats (7). However, there is no direct structural connection between the cerebellum and immune system. Therefore, it is important to examine the pathways involved in cerebellar immunomodulation in order to better understand the mechanisms of PSD.

It is well known that the hypothalamus is a crucial immunoregulatory center, which modulates the immune system via the sympathetic nervous system and the hypothalamic-pituitary-adrenal axis (11-13). A direct cerebellar-hypothalamic projection has been found $(14,15)$, which arises from cerebellar FN neurons and mainly travels along the superior cerebellar peduncle (scp). Following crossing at the decussation of the scp (XSCP), it travels along the contralateral scp and terminates in the lateral hypothalamic area (LHA) $(14,15)$. Experiments have indicated that there are direct glutamatergic and $\gamma$-aminobutyric acid (GABA)ergic projections from the FN to the LHA, which convey immunomodulating information from the FN $(16,17)$. Accordingly, cerebellar immunoregulatory information may be transmitted to the hypothalamus via direct cerebellar-hypothalamic projections. Based on these findings, it was hypothesized in the present study that direct cerebellar-hypothalamic projections may transmit cerebellar immunoregulatory information to the hypothalamus, through which the regulatory information is then conveyed to the immune system involved in PSD.

In the present study, lesions of the FN or XSCP, behavioral signs of depression, mRNA levels of tumor necrosis factor (TNF)- $\alpha$, interleukin (IL)-6, and IL-1 $\beta$ in the hippocampus, and the content of GABA and glutamate in the LHA were examined. The aim was to provide further evidence of the mechanisms suggested for the involvement of the cerebellum in PSD.

\section{Materials and methods}

Establishment of a PSD rat model. Healthy Sprague-Dawley (SD) rats weighing 240-260 g, which were aged 13 weeks, were provided by the Center of Experimental Animals, Jinzhou Medical University (Jinzhou, China). A total of 30 SD rats were randomly divided into five groups: Sham-operated, Stroke, PSD, FN lesion, and XSCP lesion groups. The middle cerebral artery was not occluded in animals of the Sham group, whereas the rats in the Stroke, PSD, FN lesion, and XSCP lesion groups underwent middle cerebral artery occlusion (MCAO). The rats were housed $(n=5 /$ cage) in quiet a room that was maintained at $21-22^{\circ} \mathrm{C}$ with $50 \%$ relative humidity and a 12/12-h light/dark cycle. They were allowed free access to food and water. The rats in the PSD group were subjected to isolated housing in combination with chronic and unexpected mild stress (CUMS) comprising water deprivation, wet litter, food deprivation, $45^{\circ}$ cage tilt, overnight lighting, behavioral restriction, tail clamping, electric shock to foot, and ice-water swimming, in order to set up a depression model. The protocol was approved by Jinzhou Medical University for the Care and Use of Laboratory Animals.

Induction of bilateral cerebellar FN lesions using KA. The KA (Sigma, EMD Millipore, Billerica, MA, USA) was dissolved in saline. Following the administration of pentobarbital (55 mg/kg, i.p.), the MCAO rats were placed in a stereotaxic apparatus (David Kopf 902-A; David Kopf Instruments, Tujunga, CA, USA). The KA (0.4 $\mu \mathrm{g}$ in $0.4 \mu \mathrm{l}$ sterilized saline) was infused into each lateral FN using the following stereotaxic coordinates: $11.5 \mathrm{~mm}$ posterior to the bregma, $1.1 \mathrm{~mm}$ left/right of the midline, and $6.3 \mathrm{~mm}$ ventral to the bregma (18). The infusion was made over $2 \mathrm{~min}$ and the needle was then left in place for $3 \mathrm{~min}$. Data on body weight, consumption of sucrose water, rearing activity, locomotor activity, and GABA and glutamate of all rats were collected during the experiment.

Induction of XSCP lesions using an electrode. Anesthesia and placement were performed as described above. Electrolytic lesions were produced using a stainless steel electrode insulated within $0.1 \mathrm{~mm}$ of its sharpened tip. The electrode was inserted into the XSCP using the following stereotaxic coordinates: $6.7-8.0 \mathrm{~mm}$ posterior to the bregma, $0 \mathrm{~mm}$ left/right of the midline, and 7.8-8.0 mm ventral to the bregma (18). A current of $0.5 \mathrm{~mA}$ was applied and the duration was $10 \mathrm{sec}$. Data were collected as above.

Open-field test (OFT). The rats were placed in the front right corner of a clear acrylic box (16x16) for $20 \mathrm{~min}$. A computer operated PAS Open Field system (San Diego Instruments, San Diego, CA, USA) was used in accordance with the manufacturer's protocol to quantify the locomotor and rearing activity as the total number of beam breaks.

Dialysate collection and high-performance liquid chromatography (HPLC). The contents of glutamate and GABA were measured by HPLC. Anesthesia and placement were performed as described above. The microdialysis probes (CMA Microdialysis AB, Kista, Sweden) were inserted into the LHA using the following stereotaxic coordinates: -1.8-2.56 $\mathrm{mm}$ posterior to the bregma, 1.4-2.6 $\mathrm{mm}$ right of the midline, and 7.6-8.4 $\mathrm{mm}$ ventral to the bregma (18). The probes were perfused with an artificial cerebrospinal fluid at a flow rate of $2 \mu \mathrm{l} / \mathrm{min}$. Following implantation, the dialysate fractions were collected at 30-min intervals. The fractions collected in the first $1 \mathrm{~h}$ were discarded to avoid parenchymal disturbance prior to a semi-steady-state level being reached. The subsequent two fractions were used for evaluation. The dialysates were automatically collected with a refrigerated autosampler (CMA Microdialysis $\mathrm{AB}$ ) and stored at $-80^{\circ} \mathrm{C}$ until analysis.

The dialysate samples were individually homogenized in $0.25 \mathrm{ml}$ methanol and centrifuged for $10 \mathrm{~min}(16,873 \mathrm{x} \mathrm{g})$ at $4^{\circ} \mathrm{C}$. Briefly, $20 \mu \mathrm{l}$ supernatant was injected into an HPLC machine equipped with a fluorescent detector (Waters Corporation, Milford, MA, USA) and an amino acid analysis column (Waters Corporation). Separation of the amino acid mixture was performed using a gradient system at a flow rate of $1 \mathrm{ml} / \mathrm{min}$. Mobile phase A contained a sodium acetate buffer and mobile phase B contained acetonitrile. Fluorometric detection was performed at an excitation and emission wavelength of 250 and $395 \mathrm{~nm}$, respectively.

Optical microscopy. At the end of the experiment, the animals were sacrificed by decapitation. The brain tissues were removed 
Table I. Primer sequences for reverse transcription-quantitative polymerase chain reaction analysis.

Primer (5'-3')

\begin{tabular}{lll}
\cline { 2 - 3 } Gene & \multicolumn{1}{c}{ Forward } & \multicolumn{1}{c}{ Reverse } \\
\hline IL-1 $\beta$ & TGCAGAGTTCCCCAACTGGTACA & GTGCTGCCTAATGTCCCCTTG \\
IL-6 & CCGGAGAGGAGACTTCACAG & TCCACGATTTCCCAGAGAAC \\
TNF- $\alpha$ & TCAGCCTCTTCTCATTCCTG & TGAAGAGAACCTGGGAGTAG \\
$\beta$-actin & GATCCGTGAAGATCAAGATCATTGCT & TGATCTTCATTTTTTACGCGTGAATT \\
\hline
\end{tabular}

IL, interleukin; TNF, tumor necrosis factor.

and fixed with $4 \%$ paraformaldehyde in $0.1 \mathrm{M}$ phosphate buffer ( $\mathrm{pH} 7.4$ ) for $48 \mathrm{~h}$. Subsequently, the brain tissues were sectioned coronally into 30-mm-thick sections at the FN, XSCP and LHA levels. Nissl staining of the sections was performed to observe the locations of the lesions and microdialysis probes under an optical microscope at a magnification of $\mathrm{x} 200$.

$R N A$ isolation and reverse transcription-quantitative polymerase chain reaction ( $R T-q P C R)$ analysis. TRIzol reagent (Invitrogen, Thermo Fisher Scientific, Inc., Waltham, MA, USA) was used to extract the total RNA from the tissue samples according to the manufacturer's protocol. RT-qPCR was used to evaluate the mRNA levels of TNF- $\alpha$, IL- 6 , and IL-1 $\beta$. Superscript II Reverse Transcriptase (Invitrogen, Thermo Fisher Scientific, Inc.) was used to reverse transcribe the isolated RNA into cDNA using an oligo dT18 primer in accordance with the manufacturer's protocol. The Sequence Detection System 7900HT containing the Universal Master Mix (both Applied Biosystems, Thermo Fisher Scientific, Inc.) was used to perform the qPCR with specific primers (Table I). The PCR procedure started with an initial step of $50^{\circ} \mathrm{C}$ for $2 \mathrm{~min}$, then $95^{\circ} \mathrm{C}$ for $2 \mathrm{~min}$, followed by 40 cycles of denaturation at $94^{\circ} \mathrm{C}$ for $15 \mathrm{sec}$, annealing at $58-62^{\circ} \mathrm{C}$ for $15 \mathrm{sec}$ and extension at $72^{\circ} \mathrm{C}$ for $15 \mathrm{sec}$. Melt curve analyses $\left(65-95^{\circ} \mathrm{C}\right.$ at a rate of $0.1^{\circ} \mathrm{C}$ per sec) were performed at the end of each PCR. If multiple peaks were observed, the data were not used. Each threshold cycle $(\mathrm{Ct})$ value was calculated by taking an average of values obtained from triplicates samples. $\beta$-actin was used as the internal control for normalization of the mRNA expression of TNF- $\alpha$ IL-6, and IL-1 $\beta$. The $2^{-\Delta \Delta C q}$ method (19) was used to calculate expression of the cytokines.

Statistical analysis. All data are presented as the mean \pm standard deviation. SPSS 21.0 software (IBM SPSS, Armonk, NY, USA) was used for statistical analysis. The difference among multiple experimental groups was detected by one-way analysis of variance. Variance was determined using the Least-Significant Difference t-test and Student-Newman-Keuls post hoc test. $\mathrm{P}<0.05$ was considered to indicate a statistically significant difference.

\section{Results}

Histopathology of the FN, XSCP and LHA lesions. In terms of the Nissl-stained cerebellar sections at 1, 4, 8, 12, 16, 20,
24 and 28 days post-injection, all animals receiving KA injections showed a marked loss of Nissl bodies, indicating that the neuronal bodies in the FN had been destroyed. The interposed nucleus adjacent to the destroyed FN did not exhibit notable neuronal loss (Fig. 1A). The electrolytic currents applied in the experiment resulted in acute spherical lesions centered on the tip of the electrode. The acute electrolytic lesions were characterized by a central zone of cavitation injury, surrounded by a sphere of carbonized tissues, which showed that the region of XSCP had been destroyed (Fig. 1B). It was also noted that hemorrhagic lesions were present around the microdialysis probe track, which confirmed the correct implantation of the microdialysis probe in the parenchyma of the LHA (Fig. 1C).

Establishment of a rat model of post-stoke depression. The healthy SD rats were subjected to MACO. The rats with PSD, FN, and XSCP lesions were further subjected to CUMS to develop a rat depression model. The FN lesion and XSCP lesion groups were established as described above.

FN and XSCP lesions induce depressive-like behaviors. In the PSD group, the rats exhibited depressive-like behaviors, including body weight loss (Fig. 2), decreased sucrose preference (Fig. 3), decreased locomotor activities (Fig. 4) and decreased rearing activities (Fig. 5) in the OFT. Similarly, the rats with FN lesions exhibited significantly decreased body weight, sucrose preference, locomotor activities and rearing activities in the OFT. The rats in the XSCP lesion group also exhibited decreased body weight, sucrose preference, locomotor activities and rearing activities in the OFT. These data indicated that FN lesions or XSCP lesions led to depressive-like behaviors in the PSD rat models.

FN and XSCP lesions increase the expression of inflammatory cytokines. Significantly higher levels of inflammatory markers are associated with a range of depressive symptoms. In the present study, the mRNA levels of TNF- $\alpha$, IL- 6 , and IL- $1 \beta$ in hippocampal tissues were examined (Fig. 6). Compared with the sham rats, transient upregulation of these cytokines was observed in the stroke rats on days 7 and 14. The PSD rats exhibited significantly upregulated cytokine expression between days 7 and 21 . The stroke rats with the FN or XSCP lesions exhibited significantly higher levels of cytokines, consistent with the increase in the depressive symptoms of the FN and XSCP rats. 


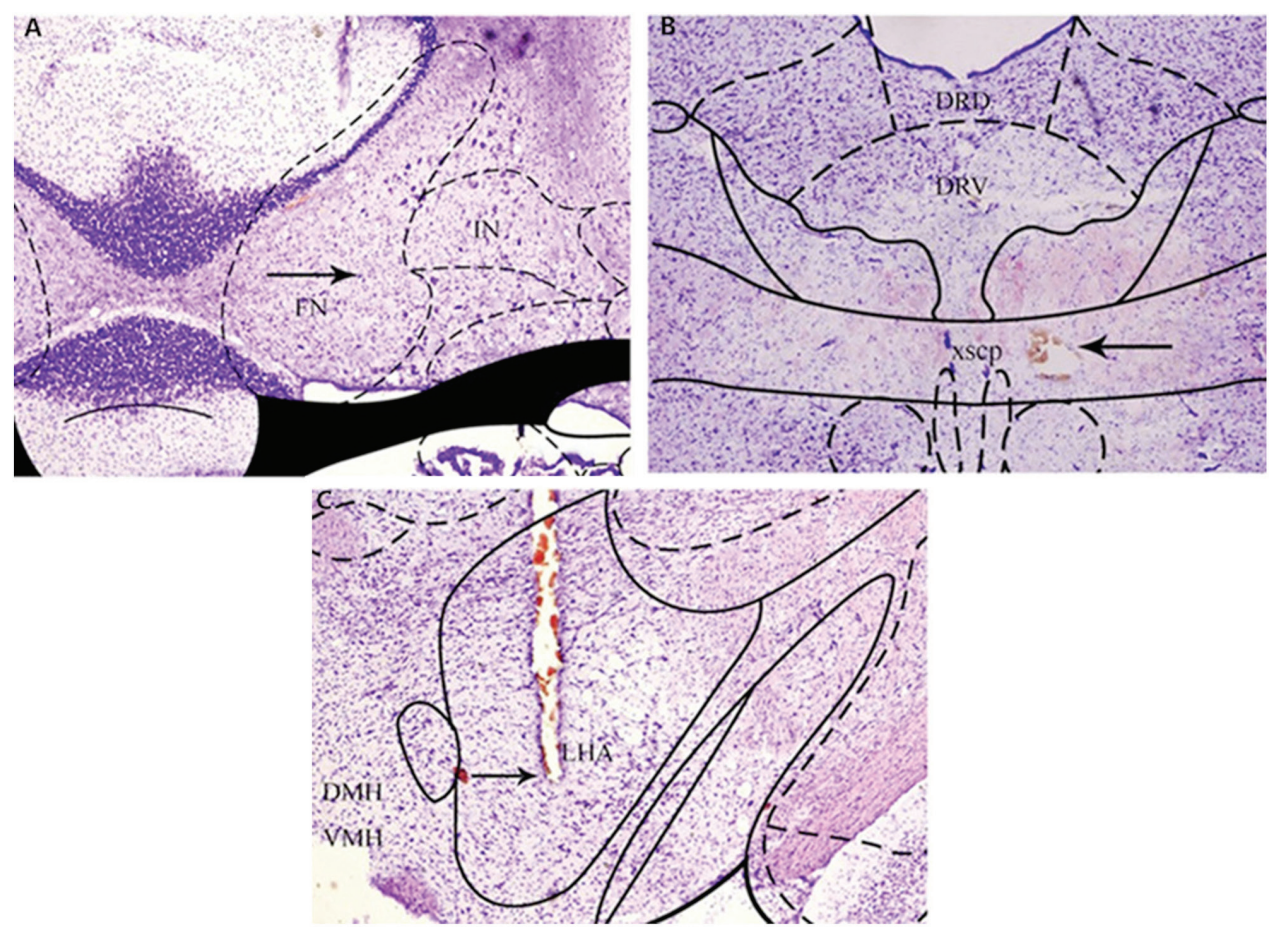

Figure 1. Images of FN, XSCP and LHA lesions in Nissl-stained sections. The sections were $30 \mu \mathrm{m}$ in thickness and were stained with cresyl violet acetate. (A) Significant neuronal loss was present in the FN, with preservation of neurons in the adjacent IN. (B) Round cavitation injury was present in the XSCP. (C) Hemorrhagic lesions were present around the microdialysis probe track in the LHA. The arrows point to lesions. FN, fastigial nucleus; XSCP, decussation of the superior cerebellar peduncle; LHA, lateral hypothalamic area; DRD, dorsal raphe nucleus, dorsal region; DRV, dorsal raphe nucleus, ventral region; $\mathrm{DMH}$, dorsomedial hypothalamic nucleus; VMH, ventromedial hypothalamic nucleus.

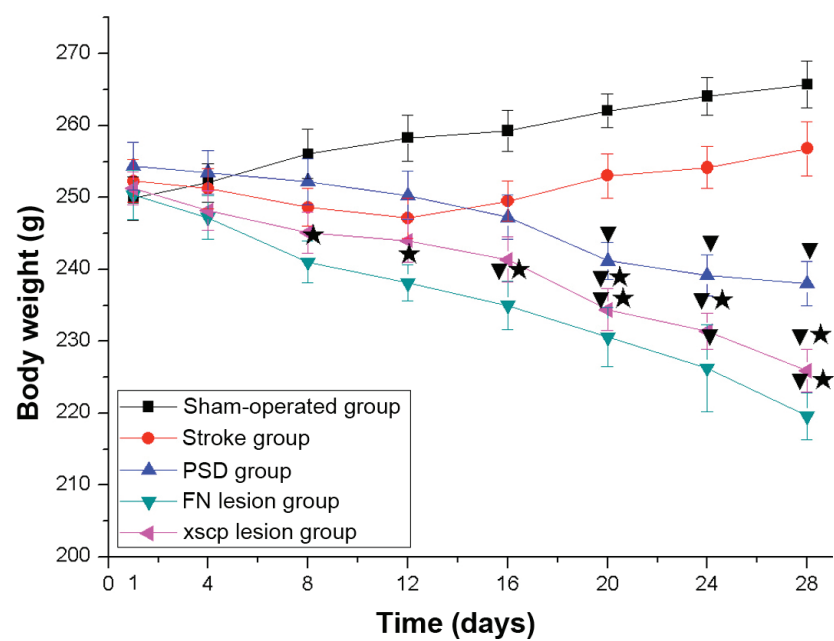

Figure 2. Dynamic changes in rat body weight. Rat body weight in the Stroke group reduced until day 12 , and increased subsequently. The body weight in the PSD, FN lesion and XSCP lesion groups continually decreased and reached their lowest level on day 28 . $^{\top} \mathrm{P}<0.01$, compared with the Stroke group, ${ }^{\star} \mathrm{P}<0.05$, compared with the PSD lesion group. The error bars represent the standard deviation $(\mathrm{n}=6)$. PSD, post-stroke depression; FN, fastigial nucleus; XSCP, decussation of the superior cerebellar peduncle.

FN and XSCP lesions decrease the content of GABA and glutamate in the LHA. The content of GABA and glutamate in the LHA is tightly coupled to the change in the expression of inflammatory cytokines $(16,17)$. The present study also examined whether FN and XSCP lesions affected the content of GABA and glutamate. The results indicated significant loss of GABA (Fig. 7) and glutamate (Fig. 8) content in the

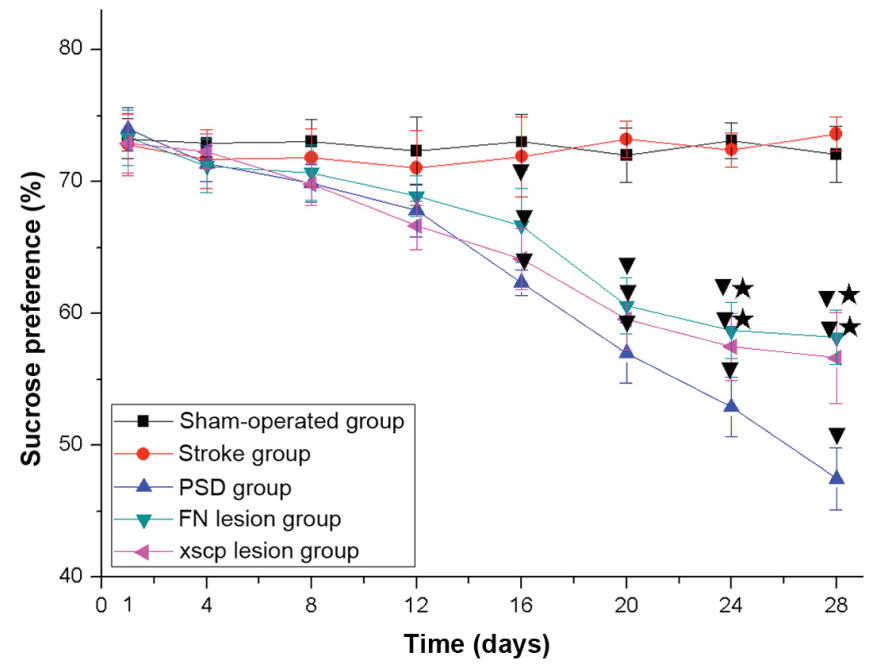

Figure 3. Dynamic changes in sucrose preference. The consumption of sucrose water decreased in the PSD, FN lesion and XSCP lesion groups during the first 24 days, compared with that in the Sham-operated and Stroke groups. Subsequently, the consumption of sucrose water in the PSD group decreased significantly and reached the lowest point on day 28 . The sucrose consumption in the FN lesion and XSCP lesion groups gradually decreased and remained stable from day 20. $\mathbf{P}<0.01$, compared with the Stroke group; ${ }^{\star} \mathrm{P}<0.01$, compared with the PSD group. The error bars represent the standard deviation ( $n=6$ ). PSD, post-stroke depression; FN, fastigial nucleus; XSCP, decussation of the superior cerebellar peduncle.

LHA of that the rats in the PSD group. In addition, FN and XSCP lesions significantly decreased the content of GABA and glutamate. 


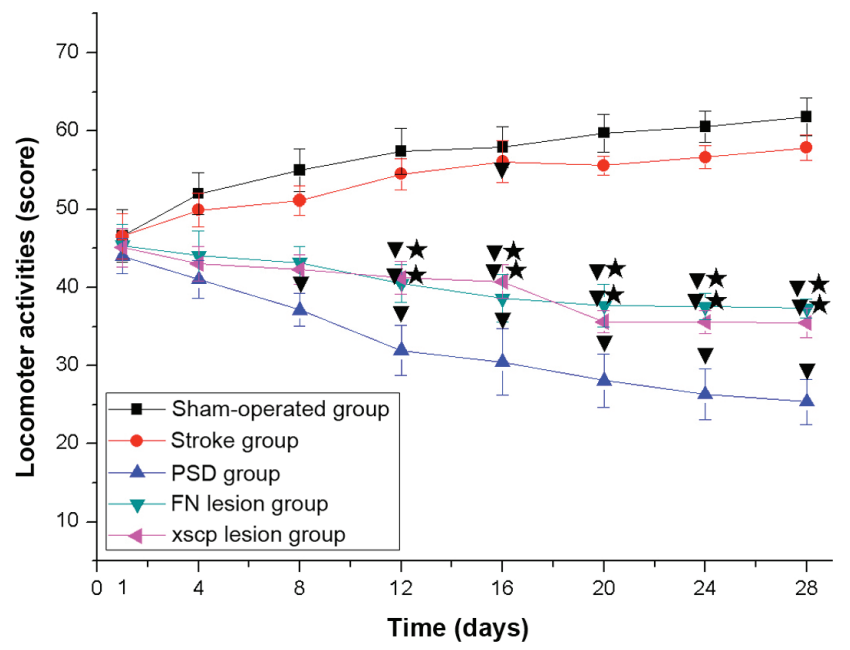

Figure 4. Dynamic changes in locomotor activity during the open-field test. There was no significant difference in the scores for locomotor activity between the Sham-operated and Stroke groups. Locomotor activity in the PSD group was significantly reduced compared with that in the Stroke group and reached its lowest point on day 28. Locomotor activity in the FN lesion and XSCP lesion groups decreased until day 20 and remained constant thereafter. The difference between the FN lesion and XSCP lesion groups was statistically significant. ${ }^{\top} \mathrm{P}<0.01$, compared with the Stroke group; ${ }^{\star} \mathrm{P}<0.05$, compared with the PSD group. The error bars represent the standard deviation $(\mathrm{n}=6)$. PSD, post-stroke depression; FN, fastigial nucleus; XSCP, decussation of the superior cerebellar peduncle.

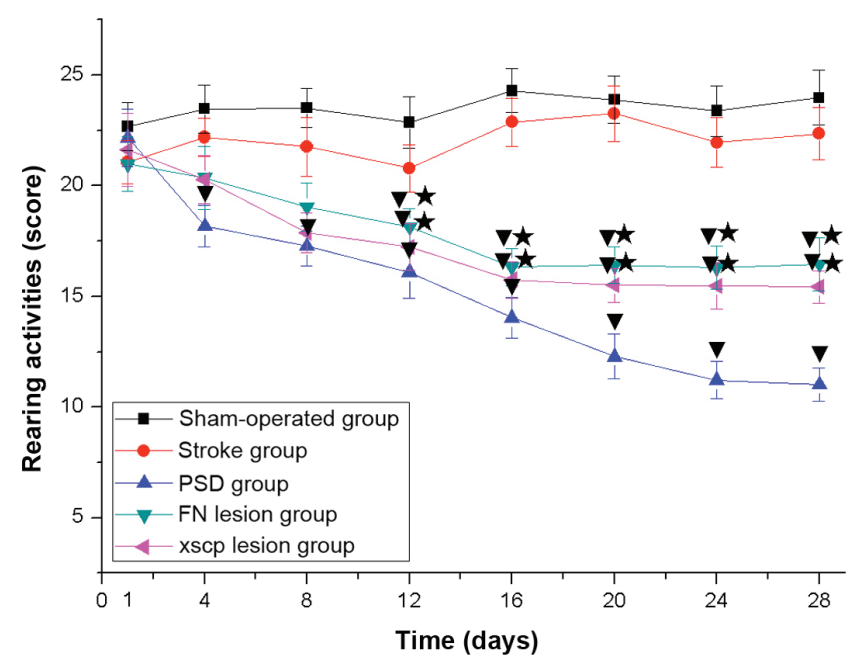

Figure 5. Dynamic changes in rearing activity of the open-field test. There was no significant difference in the scores for rearing activity between the Sham-operated and Stroke groups. Rearing activity in the PSD group was significantly reduced compared with that in the Stroke group and reached its lowest point on day 28. Rearing activity in the FN lesion and XSCP lesion groups decreased until day 16 and remained constant thereafter. The difference between the FN lesion and XSCP lesion groups was statistically significant. ${ }^{\mathrm{P}} \mathrm{P}<0.01$, compared with the Stroke group; ${ }^{\star} \mathrm{P}<0.05$, compared with the PSD group. The error bars represent standard deviation $(n=6)$. PSD, post-stroke depression; FN, fastigial nucleus; XSCP, decussation of the superior cerebellar peduncle.

\section{Discussion}

Based on previous results, MCAO and CUMS were induced in rats to mimic post-stroke depressive-like symptoms in patients with PSD, which mainly include the following: i) Reduced preference for sucrose water and a putative indicator of anhedonia (20); ii) decreased locomotor and rearing activities suggesting changes in incentive motivation and emotionality (21); iii) body weight loss similar to that observed in PSD (22). In the present study, the results showed that MCAO and CUMS decreased body weight and sucrose preference in addition to locomotor and rearing activities in the OFT.

As there are no KA receptors on neuronal axons, KA has been reported to destroy neuron bodies only, without damaging nerve fibers across the neural nuclei (23). In the present study, it was observed that Nissl bodies, which are present in the plasma of normal neuronal bodies and are closely associated with neuronal degeneration and death, had almost all disappeared in the FN infused with KA. These changes demonstrated that KA effectively destroyed the neuronal bodies in the cerebellar FN. In addition, the results showed that the rats in the FN lesion group exhibited similar behavioral profiles as those observed in the PSD group during the whole 28-day period. Cerebellar FN involvement was suggested in previous studies, which showed that the stimulation or damage of the FN in animals or patients ameliorated or provoked depression $(7,24,25)$. Therefore, the findings of the present study support the hypothesis that the cerebellar FN is involved in the regulation of depression. However, how the cerebellar FN functions in PSD remains to be fully elucidated.

The cerebellar FN is closely associated with inflammation. Previously, it was reported that stimulation of the cerebellar FN can inhibit cerebrovascular inflammation (9). Our preliminary study also showed that FNS reduced the expression of inflammatory cytokines in the hippocampus (7). In addition, FN lesions can gradually promote the function of $\mathrm{T}$ cells, B cells and natural killer cells over time (10). Therefore, in terms of the inflammatory mechanisms underlying PSD (2), the mRNA expression levels of inflammatory cytokines, including TNF- $\alpha$, IL- 6 , and IL-1 $\beta$, in the hippocampus of rats were measured in the present study. The results showed that the mRNA expression levels of TNF- $\alpha$, IL- 6 , and IL- $1 \beta$ were increased in the PSD and FN lesion groups. These results suggested that the involvement of the cerebellar FN in PSD may be achieved via mediating inflammatory cytokines.

As there are no direct cerebellar innervations to lymphoid organs, cerebellar immunomodulation must be conveyed through other pathways. Due to its direct connection with the cerebellum and its predominant neuroimmunomodulatory effect, the hypothalamus may be one of the most likely candidates in transmitting immunoregulatory information from the cerebellum to the lymphoid organs $(14,15,26-28)$. Previously, it was demonstrated that there were direct glutamatergic and GABAergic projections between the FN and LHA, which conveyed immunomodulating information from the FN $(16,17)$. In the present study, an electrolytic method was used to destroy the projections from cerebellar FN neurons to the hypothalamus in the XSCP lesion group, and a spherical lesion centered on the tip of the electrode showed that the XSCP region was destroyed. The present study is the first, to the best of our knowledge, to demonstrate decreases in sucrose water consumption, body weight and rat activities when the mRNA levels of TNF- $\alpha$, IL- 6 , and IL- $1 \beta$ were significantly increased following the formation of XSCP lesions. In addition, the results 

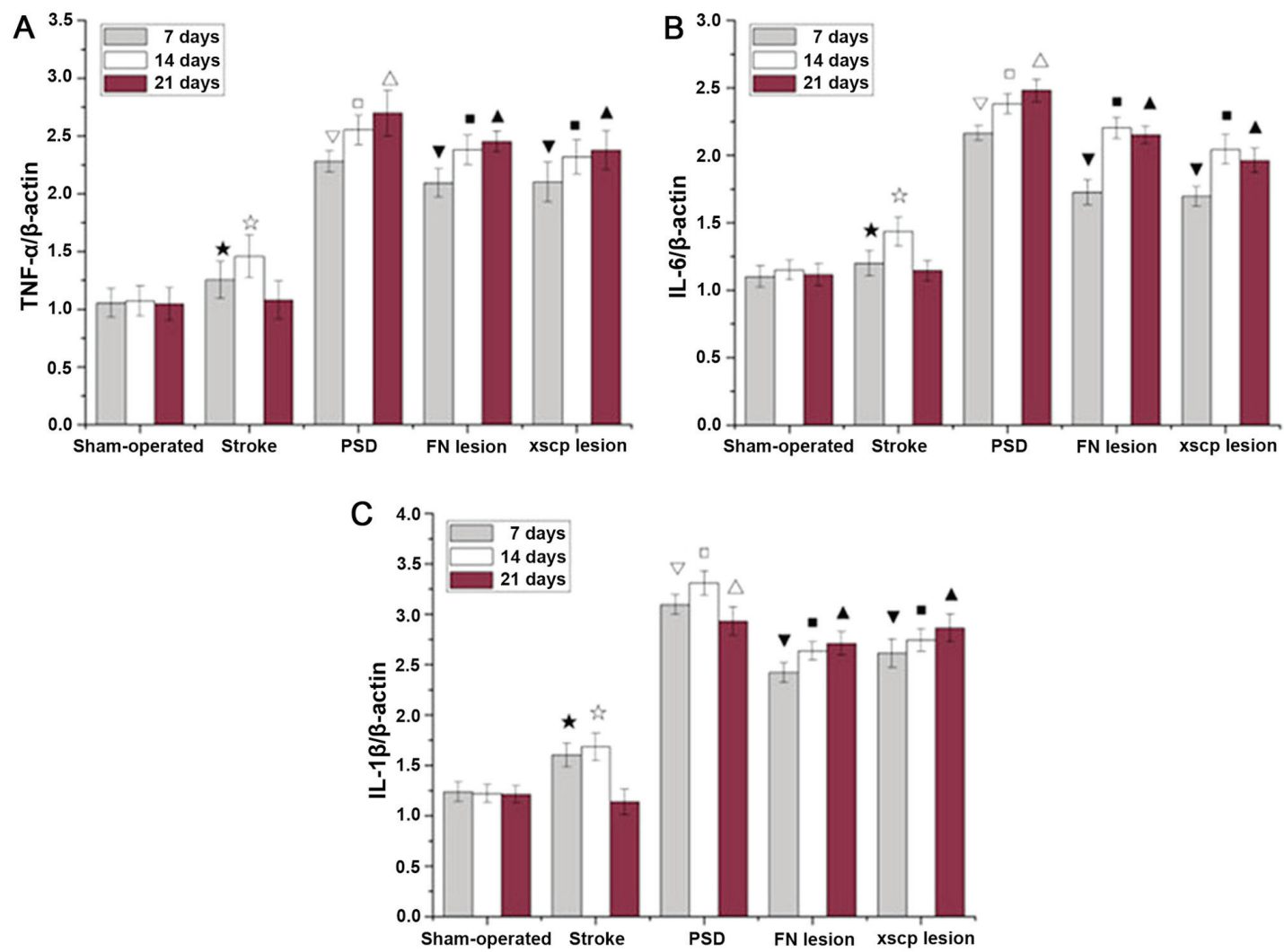

Figure 6. Effect of FN lesions or XSCP lesions on the expression of TNF- $\alpha$, IL-6, and IL-1 $\beta$ in hippocampal tissues. mRNA expression levels of (A) TNF- $\alpha$, (B) IL-6, and (C) IL-1 $\beta$ in hippocampal tissues of PSD rats on day 7, 14, and 21 are shown. Compared with the Sham-operated group, the mRNA levels of cytokines in the Stroke group increased within 14 days and then decreased to their lowest levels on day 21. Compared with the stroke group, the mRNA levels of cytokines in the PSD group were significantly increased within 14 days and then remained almost stable from day 14. In the FN lesion and XSCP lesion groups, the corresponding mRNA levels showed a similar trend to that in the PSD group, although their absolute mRNA levels were marginally lower than those in the PSD group. ${ }^{\star} \mathrm{P}<0.05$, compared with the Sham group on day 7 ; ${ }^{\circledR} \mathrm{P}<0.05$, compared with the Sham group on day 14 ; ${ }^{\nabla} \mathrm{P}<0.01$, compared with the Stroke group on day 7; ${ }^{\square} \mathrm{P}<0.01$, compared with the Stroke group on day $14 ;{ }^{\wedge} \mathrm{P}<0.01$, compared with the Stroke group on day $21 ;{ }^{\vee} \mathrm{P}<0.05$, compared with the PSD group on day $7 ; \cdot \mathrm{P}<0.05$, compared with the PSD group on day $14 ;{ }^{\wedge} \mathrm{P}<0.05$, compared with the PSD group on day 21 . The error bars represent the standard deviation $(\mathrm{n}=6)$. PSD, post-stroke depression; FN, fastigial nucleus; XSCP, decussation of the superior cerebellar peduncle; TNF- $\alpha$, tumor necrosis factor- $\alpha$; IL, interleukin.

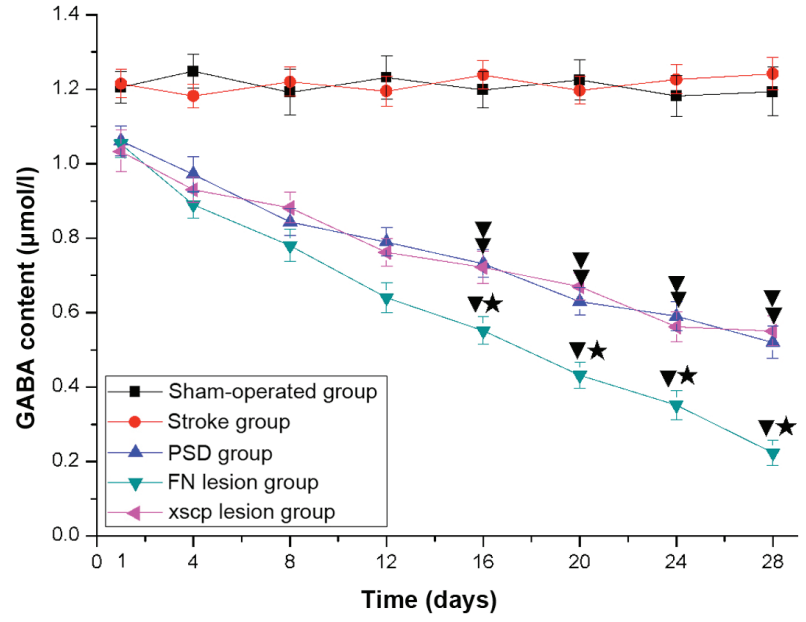

Figure 7. Dynamic changes of GABA in the LHA. There was no significant difference in the content of GABA between the sham-operated and stroke groups. The content of GABA in the PSD group was significantly decreased compared with that in the Stroke group, reaching its lowest point on day 28. In the XSCP group, the content of GABA showed a similar trend to that in the PSD group. The GABA content in the LHA had almost disappeared in the FN lesion group on day 28. ${ }^{\top} \mathrm{P}<0.01$, compared with the Stroke group; ${ }^{\star} \mathrm{P}<0.05$, compared with the PSD group. The error bars represent the standard deviation $(n=6)$. LHA, lateral hypothalamic area; PSD, post-stroke depression; FN, fastigial nucleus; XSCP, decussation of the superior cerebellar peduncle; GABA, $\gamma$-aminobutyric acid.

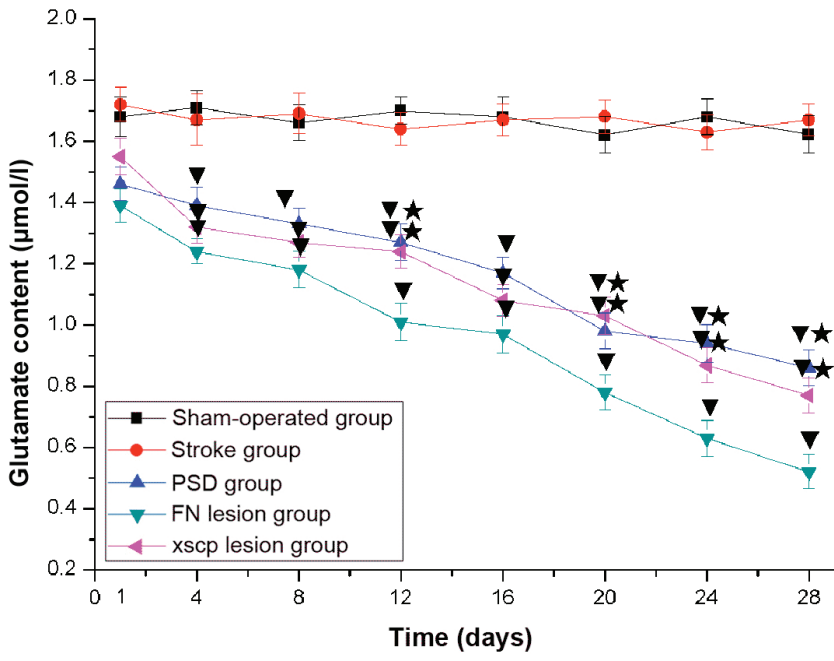

Figure 8. Dynamic changes of glutamate in the lateral hypothalamic area. There was no significant difference in the content of glutamate between the Sham-operated and Stroke groups. The content of glutamate in the PSD, FN lesion and XSCP lesion groups was significantly decreased compared with that in the Stroke group, until its lowest point was reached on day 28 . ${ }^{\top} \mathrm{P}<0.01$, compared with the Stroke group; ${ }^{\star} \mathrm{P}<0.05$, compared with the PSD group. The error bars represent the standard deviation $(n=6)$. PSD, post-stroke depression; FN, fastigial nucleus; XSCP, decussation of the superior cerebellar peduncle. 
suggested that the direct cerebellar-hypothalamic projections may act as a potential pathway to transmit immunoregulatory information from the cerebellar FN in PSD.

By the anterograde and retrograde tracing of nerve tracts in conjunction with GABA and glutamate immunohistochemistry, previous studies have found that the direct cerebellar-hypothalamic projections mainly contain glutamatergic and GABAergic nerve fibers $(15,16)$. Electrophysiological experiments have also revealed that stimulation of the $\mathrm{FN}$ evokes monosynaptic inhibitory and polysynaptic excitatory responses of LHA neurons, suggesting that there is a direct cerebellar-hypothalamic GABAergic and glutamatergic projection between the cerebellum and the hypothalamus $(26,27,29)$. In the present study, the glutamate and GABA contents in the LHA were decreased in the FN lesion group, indicating that the FN lesions decreased FN GABAergic and glutamatergic transmission to the hypothalamus. Of note, XSCP lesions also attenuated the GABA and glutamate content in the LHA. The similar effect of FN and XSCP lesions on the glutamate and GABA contents in the LHA showed that the direct cerebellar-hypothalamic projection released GABA and glutamate as neurotransmitters. Therefore, it was hypothesized that the decrease of GABA and glutamate content in the LHA increased the mRNA expression levels of TNF- $\alpha$, IL-6, and IL-1 $\beta$, suggesting that the immune response was modulated by cerebellar FN GABAergic and glutamatergic neurons. Previous reports $(16,17)$ have suggested that GABAergic and glutamatergic neurons in the cerebellar $\mathrm{FN}$ regulated innate and adaptive immune responses, consistent with the results of the present study.

In conclusion, FN and XSCP lesions resulted in decreased levels of glutamate and GABA in the LHA, induced the amplification of genes corresponding to inflammatory cytokines, and ultimately caused depression-like behaviors. These findings revealed that FN glutamatergic and GABAergic projections to the LHA may be significant in PSD. In addition, the results suggested that the cerebellar FN regulated mood and emotion via the direct cerebellar-hypothalamic GABAergic and glutamatergic projections (29).

\section{Acknowledgements}

Not applicable.

\section{Funding}

The present study was sponsored by the National Science Foundation of China (grant nos. 81371461 and 81241050) and the National Science Foundation of Liaoning Province (grant no. 2013022018).

\section{Availability of data and materials}

The datasets used and analyzed during the current study are available from the corresponding author on reasonable request.

\section{Authors' contributions}

YL designed the study and wrote the manuscript. XZ and BY were peformed experiments and collected data. LC performed the statistical analysis. RS designed the study, supervised the study and revised the manuscript.

\section{Ethics approval and consent to participate}

All animal experiments complied with the Guidelines on Ethical Standards (30). The current study was approved by Jinzhou Medical University Ethics Committee for the Care and Use of Laboratory Animals.

\section{Patient consent for publication}

Not applicable.

\section{Competing interests}

The authors declare that they have no competing interests.

\section{References}

1. Towfighi A, Ovbiagele B, El Husseini N, Hackett ML, Jorge RE, Kissela BM, Mitchell PH, Skolarus LE, Whooley MA, Williams LS, et al: Poststroke depression: A scientific statement for healthcare professionals from the American heart association/American stroke association. Stroke 48: e30-e43, 2017.

2. Spalletta G, Bossù $P$, Ciaramella $A$, Bria $P$, Caltagirone $C$ and Robinson RG: The etiology of poststroke depression: A review of the literature and a new hypothesis involving inflammatory cytokines. Mol Psychiatry 11: 984-991, 2006.

3. Jiao JT, Cheng C, Ma YJ, Huang J, Dai MC, Jiang C, Wang C and Shao JF: Association between inflammatory cytokines and the risk of post-stroke depression, and the effect of depression on outcomes of patients with ischemic stroke in a 2-year prospective study. Exp Ther Med 12: 1591-1598, 2016.

4. Schmahmann JD and Sherman JC: The cerebellar cognitive affective syndrome. Brain 121: 561-579, 1998.

5. Bugalho P, Correa B and Viana-Baptista M: Role of the cerebellum in cognitive and behavioural control: Scientific basis and investigation models. Acta Med Port 19: 257-267, 2006 (In Portuguese).

6. Schmahmann JD, MacMore J and Vangel M: Cerebellar stroke without motor deficit: Clinical evidence for motor and non-motor domains within the human cerebellum. Neuroscience 162: 852-861, 2009.

7. Lei Z, Zhao M and Sui RB: Cerebellar fastigial nucleus electrical stimulation alleviates depressive-like behaviors in post-stroke depression rat model and potential mechanisms. Cell Physiol Biochem 41: 1403-1412, 2017.

8. Su CY, Wuang YP, Lin Y and Su JH: The role of processing speed in post-stroke cognitive dysfunction. Arch Clin Neuropsychol 30: 148-160, 2015.

9. Galea E, Glickstein SB, Feinstein DL, Golanov EV and Reis DJ: Stimulation of cerebellar fastigial nucleus inhibits interleukin-1beta-induced cerebrovascular inflammation. Am J Physiol 275: H2053-H2063, 1998.

10. Ni SJ, Qiu YH, Lu JH, Cao BB and Peng YP: Effect of cerebellar fastigial nuclear lesions on differentiation and function of thymocytes. J Neuroimmunol 222: 40-47, 2010.

11. Madden KS: Catecholamines, sympathetic innervation, and immunity. Brain Behav Immun 17 (Suppl 1): S5-S10, 2003.

12. Nance DM and Sanders VM: Autonomic innervation and regulation of the immune system (1987-2007). Brain Behav Immun 21: 736-745, 2007.

13. Trotter RN, Stornetta RL, Guyenet PG and Roberts MR: Transneuronal mapping of the CNS network controlling sympathetic outflow to the rat thymus. Auton Neurosci 131: 9-20, 2007.

14. Dietrichs E, Haines DE, Røste GK and Røste LS: Hypothalamocerebellar and cerebellohypothalamic projections-circuits for regulating nonsomatic cerebellar activity? Histol Histopathol 9: 603-614, 1994.

15. Haines DE, Dietrichs E, Mihailoff GA and McDonald EF: The cerebellar-hypothalamic axis: Basic circuits and clinical observations. Int Rev Neurobiol 41: 83-107, 1997. 
16. Cao BB, Huang Y, Jiang YY, Qiu YH and Peng YP: Cerebellar fastigial nuclear glutamatergic neurons regulate immune function via hypothalamic and sympathetic pathways. J Neuroimmune Pharmacol 10: 162-178, 2015.

17. Cao BB, Huang Y, Lu JH, Xu FF, Qiu YH and Peng YP: Cerebellar fastigial nuclear GABAergic projections to the hypothalamus modulate immune function. Brain Behav Immun 27 80-90, 2013.

18. Paxinos $\mathrm{G}$ and Watson $\mathrm{C}$ : The rat brain in stereotaxic coordinates. New York: Academic, 1986.

19. Livak KJ and Schmittgen TD: Analysis of relative gene expression data using real-time quantitative PCR and the 2(-Delta Delta C(T)) method. Methods 25: 402-408, 2001.

20. Willner P, Muscat R and Papp M: Chronic mild stress-induced anhedonia: A realistic animal model of depression. Neurosci Biobehav Rev 16: 525-534, 1992.

21. Roth KA and Katz RJ: Stress, behavioral arousal, and open field activity-a reexamination of emotionality in the rat. Neurosci Biobehav Rev 3: 247-263, 1979.

22. Sapolsky RM, Romero LM and Munck AU: How do glucocorticoids influence stress responses? Integrating permissive, suppressive, stimulatory, and preparative actions. Endocr Rev 21: 55-89, 2000.
23. Köhler C and Schwarcz R: Comparison of ibotenate and kainate neurotoxicity in rat brain: A histological study. Neuroscience 8: 819-835, 1983.

24. Liu JL, Li JP and Dong WW: A clinical study of fastigial nucleus electrical stimulation on post stroke depression. J Chin J Clin Rehabilitation 7: 1926-1927, 2003.

25. Schmahmann JD: The role of the cerebellum in affect and psychosis. J Neurolinguis 13: 189-214, 2000.

26. Wang J, Pu Y and Wang T: Influences of cerebellar interpositus nucleus and fastigial nucleus on neuronal activity of lateral hypothalamic area. Sci China C Life Sci 40: 176-183, 1997.

27. Zhang YP, Ma C, Wen YQ and Wang JJ: Convergence of gastric vagal and cerebellar fastigial nuclear inputs on glycemia-sensitive neurons of lateral hypothalamic area in the rat. Neurosci Res 45: 9-16, 2003.

28. Schutter DJ and Van Honk J: The cerebellum on the rise in human emotion. Cerebellum 4: 290-294, 2005.

29. Min BI, Oomura Y and Katafuchi T: Responses of rat lateral hypothalamic neuronal activity to fastigial nucleus stimulation. J Neurophysiol 61: 1178-1184, 1989.

(i) (9) This work is licensed under a Creative Commons

cc) At 1 No ND Atribution-NonCommercial-NoDerivatives 4.0 International (CC BY-NC-ND 4.0) License. 